\title{
Boundary value problem of a nonlinear Langevin equation with two different fractional orders and impulses
}

\author{
Guotao Wang ${ }^{1}$ Lihong Zhang ${ }^{2 *}$ and Guangxing Song ${ }^{2}$
}

"Correspondence:

zhanglih149@126.com

2 Department of Mathematics, China

University of Petroleum, Qingdao,

Shandong 266555, P.R. China

Full list of author information is

available at the end of the article

\begin{abstract}
In this paper, we study a new type of a Langevin equation involving two different fractional orders and impulses. Sufficient conditions are formulated for the existence and uniqueness of solutions of the given problems.

MSC: 34A08; 34B10; 34B37; 46N10

Keywords: nonlinear fractional Langevin equation; impulse; two different fractional orders; nonlocal conditions; uniqueness; fixed point theorem
\end{abstract}

\section{Introduction}

Fractional derivatives provide an excellent tool for the description of memory and hereditary properties of various materials and processes. These characteristics of the fractional derivatives make the fractional-order models more realistic and practical than the classical integer-order models. In fact, fractional differential equations appear naturally in a number of fields such as physics, geophysics, polymer rheology, regular variation in thermodynamics, biophysics, blood flow phenomena, aerodynamics, electro-dynamics of complex medium, viscoelasticity, Bode's analysis of feedback amplifiers, capacitor theory, electrical circuits, electron-analytical chemistry, biology, control theory, fitting of experimental data, nonlinear oscillation of earthquake, the fluid-dynamic traffic model, etc. For more details and applications, we refer the reader to the books [1-3]. For some recent development on the topic, see [4-15] and the references therein.

It is well known that a Langevin equation is widely used to describe the evolution of physical phenomena in fluctuating environments [16-18]. However, for the systems in complex media, an integer-order Langevin equation does not provide the correct description of the dynamics. One of the possible generalizations of a Langevin equation is to replace the integer-order derivative by a fractional-order derivative in it. This gives rise to a fractional Langevin equation, see [19-22] and the references therein.

In 2008, Lim, Li and Teo [23] firstly introduced a new type of a Langevin equation with two different fractional orders. The solution to this new version of a fractional Langevin equation gives a fractional Gaussian process parametrized by two indices, which provides a more flexible model for fractal processes as compared with the usual one characterized by a single index. In 2009, Lim and Teo [24] discussed the fractional oscillator process with two indices. In 2010, by using the contraction mapping principle and Krasnoselskii's

C) 2012 Wang et al.; licensee Springer. This is an Open Access article distributed under the terms of the Creative Commons Attribution License (http://creativecommons.org/licenses/by/2.0), which permits unrestricted use, distribution, and reproduction in any medium, provided the original work is properly cited. 
fixed point theorem, Ahmad and Nieto [25] studied a Langevin equation involving two fractional orders with Dirichlet boundary conditions. Recently, the existence of solutions for a three-point boundary value problem of a Langevin equation with two different fractional orders has also been studied in [26].

Motivated by the above-mentioned works, in this paper, we consider the following nonlinear Langevin equation with two different fractional orders and impulses in a Banach space $E$ :

$$
\left\{\begin{array}{l}
{ }^{C} D^{\beta}\left({ }^{C} D^{\alpha}+\lambda\right) u(t)=f(t, u(t)), \quad 0<t<1,0<\alpha, \beta \leq 1, \\
\triangle u\left(t_{k}\right)=I_{k}\left(u\left(t_{k}\right)\right), \quad k=1,2, \ldots, p, \\
\triangle^{C} D^{\alpha} u\left(t_{k}\right)=I_{k}^{*}\left(u\left(t_{k}\right)\right), \quad k=1,2, \ldots, p,
\end{array}\right.
$$

with one of the following three boundary conditions:

(i) Dirichlet boundary condition: $\quad u(0)=\gamma_{1}, \quad u(1)=\gamma_{2}$;

(ii) Nonlocal Dirichlet boundary condition: $u(0)+g_{1}(u)=\gamma_{1}, \quad u(1)=\gamma_{2}$;

(iii) Nonlocal Dirichlet boundary condition: $\quad u(0)=\gamma_{1}, \quad u(1)+g_{2}(u)=\gamma_{2}$;

where ${ }^{C} D$ is the Caputo fractional derivative, $J=[0,1], f \in C(J \times E, E), I_{k}, I_{k}^{*}, g_{1}, g_{2} \in C(E, E)$, $\lambda \in \mathbb{R}, 0=t_{0}<t_{1}<\cdots<t_{k}<\cdots<t_{p}<t_{p+1}=1, J^{\prime}=J \backslash\left\{t_{1}, t_{2}, \ldots, t_{p}\right\}, \gamma_{1}, \gamma_{2} \in E, \Delta u\left(t_{k}\right)=$ $u\left(t_{k}^{+}\right)-u\left(t_{k}^{-}\right)$, where $u\left(t_{k}^{+}\right)$and $u\left(t_{k}^{-}\right)$denote the right and the left limits of $u(t)$ at $t=t_{k}(k=$ $1,2, \ldots, p)$, respectively. $\triangle{ }^{C} D^{\alpha} u\left(t_{k}\right)$ has a similar meaning for ${ }^{C} D^{\alpha} u(t)$. Let $P C(J, E)=\{u$ : $J \rightarrow E \mid u(t)$ is continuous at $t \neq t_{k}$, left continuous at $t=t_{k}$ and $u\left(t_{k}^{+}\right)$exists, $\left.k=1,2, \ldots, p\right\}$. Evidently, $P C(J, E)$ is a Banach space endowed with the sup-norm $\|\cdot\|_{P C}$.

Nonlocal conditions were initiated by Byszewski [27] when he proved the existence and uniqueness of mild and classical solutions of nonlocal Cauchy problems. Many authors since then have considered the existence and multiplicity of solutions (or positive solutions) of nonlocal problems. The recent results on nonlocal problems of fractional differential equations can be found in [29-40]. As remarked by Byszewski [28], the nonlocal condition can be more useful than the standard initial (boundary) condition to describe some physical phenomena. For example, $g_{1}(u)$ may be given by

$$
g_{1}(u)=\sum_{i=1}^{p} c_{i} u\left(\tau_{i}\right)
$$

where $c_{i}, i=1, \ldots, p$, are given constants and $0<\tau_{1}<\cdots<\tau_{p} \leq T$.

Impulsive differential equations, which provide a natural description of observed evolution processes, are regarded as important mathematical tools for the better understanding of several real world problems in applied sciences. The theory of impulsive differential equations of integer order has found its extensive applications in realistic mathematical modeling of a wide variety of practical situations and has emerged as an important area of investigation. The impulsive differential equations of fractional order have also attracted a considerable attention and a variety of results can be found in the papers [41-51].

To the best knowledge of the authors, no paper has considered nonlinear Langevin equations involving two different fractional orders and impulses, i.e., problems (2.1), (3.1) and (3.2). This paper fills this gap in the literature. 
This paper is organized as follows. In Section 2, we present some preliminary results. Consequently, problem (2.1) is reduced to an equivalent integral equation. Then, by using the fixed point theory, we study the existence and uniqueness of a Dirichlet boundary value problem for nonlinear Langevin equations involving two different fractional orders and impulses. In Section 3, we indicate some generalizations to nonlocal Dirichlet boundary value problems. The last section is devoted to an example illustrating the applicability of the imposed conditions. These results can be considered as a contribution to this emerging field.

\section{Dirichlet boundary value problem}

In this section, we consider the following Dirichlet boundary value problem:

$$
\left\{\begin{array}{l}
{ }^{C} D^{\beta}\left({ }^{C} D^{\alpha}+\lambda\right) u(t)=f(t, u(t)), \quad 0<t<1,0<\alpha, \beta \leq 1, \\
\triangle u\left(t_{k}\right)=I_{k}\left(u\left(t_{k}\right)\right), \quad k=1,2, \ldots, p, \\
\triangle^{C} D^{\alpha} u\left(t_{k}\right)=I_{k}^{*}\left(u\left(t_{k}\right)\right), \quad k=1,2, \ldots, p, \\
u(0)=\gamma_{1}, \quad u(1)=\gamma_{2} .
\end{array}\right.
$$

For the sake of convenience, we introduce the following notations:

$$
J_{0}=\left[0, t_{1}\right], \quad J_{1}=\left(t_{1}, t_{2}\right], \quad \ldots, \quad J_{p-1}=\left(t_{p-1}, t_{p}\right], \quad J_{p}=\left(t_{p}, 1\right] .
$$

Definition 2.1 A function $u \in P C(J, E)$ with its Caputo derivative of fractional order existing on $J^{\prime}$ is a solution of (2.1) if it satisfies (2.1).

Lemma 2.1 [1] Let $\alpha>0$, then the fractional differential equation

$$
{ }^{C} D^{\alpha} u(t)=0
$$

has a solution

$$
u(t)=C_{0}+C_{1} t+C_{2} t^{2}+\cdots+C_{n-1} t^{n-1}, \quad c_{i} \in \mathbb{R}, \quad i=0,1,2, \ldots, n-1, n=[\alpha]+1 .
$$

Lemma 2.2 [1] Let $\alpha>0$, then

$$
I^{\alpha C} D^{\alpha} u(t)=u(t)+C_{0}+C_{1} t+C_{2} t^{2}+\cdots+C_{n-1} t^{n-1}
$$

for some $C_{i} \in \mathbb{R}, i=1,2, \ldots, n, n=[\alpha]+1$

\subsection{Existence result}

Lemma 2.3 For any $y \in C[0,1]$, a function $u$ is a solution of the following Dirichlet boundary value problem:

$$
\left\{\begin{array}{l}
{ }^{C} D^{\beta}\left({ }^{C} D^{\alpha}+\lambda\right) u(t)=y(t), \quad 0<t<1,0<\alpha, \beta \leq 1, \\
\triangle u\left(t_{k}\right)=I_{k}\left(u\left(t_{k}\right)\right), \quad k=1,2, \ldots, p, \\
\triangle^{C} D^{\alpha} u\left(t_{k}\right)=I_{k}^{*}\left(u\left(t_{k}\right)\right), \quad k=1,2, \ldots, p, \\
u(0)=\gamma_{1}, \quad u(1)=\gamma_{2},
\end{array}\right.
$$


if and only if $u$ is a solution of the fractional integral equation

$$
u(t)=\left\{\begin{aligned}
\int_{0}^{t} & \frac{(t-s)^{\alpha-1}}{\Gamma(\alpha)} \int_{0}^{s} \frac{(s-r)^{\beta-1}}{\Gamma(\beta)} y(r) d r d s-\lambda \int_{0}^{t} \frac{(t-s)^{\alpha-1}}{\Gamma(\alpha)} u(s) d s \\
& +\frac{C t^{\alpha}}{\Gamma(\alpha+1)}+\gamma_{1}, \quad t \in J_{0} ; \\
\int_{t_{k}}^{t} & \frac{(t-s)^{\alpha-1}}{\Gamma(\alpha)} \int_{t_{k}}^{s} \frac{(s-r)^{\beta-1}}{\Gamma(\beta)} y(r) d r d s+\sum_{i=1}^{k} \int_{t_{i-1}}^{t_{i}} \frac{\left(t_{i}-\right)^{\alpha-1}}{\Gamma(\alpha)} \int_{t_{i-1}}^{s} \frac{(s-r)^{\beta-1}}{\Gamma(\beta)} y(r) d r d s \\
& +\frac{\left(t-t_{k}\right)^{\alpha}}{\Gamma(\alpha+1) \Gamma(\beta)} \sum_{i=1}^{k} \int_{t_{i-1}}^{t_{i}}\left(t_{i}-s\right)^{\beta-1} y(s) d s \\
& +\sum_{i=2}^{k} \frac{\left(t_{i}-t_{i-1}\right)^{\alpha}}{\Gamma(\alpha+1) \Gamma(\beta)} \int_{0}^{t_{1}}\left(t_{1}-s\right)^{\beta-1} y(s) d s+\cdots \\
& +\sum_{i=k-1}^{k} \frac{\left(t_{i}-t_{i-1}\right)^{\alpha}}{\Gamma(\alpha+1) \Gamma(\beta)} \int_{t_{k-3}}^{t_{k-2}}\left(t_{k-2}-s\right)^{\beta-1} y(s) d s \\
& +\frac{\left(t_{k}-t_{k-1}\right)^{\alpha}}{\Gamma(\alpha+1) \Gamma(\beta)} \int_{t_{k-2}}^{t_{k-1}}\left(t_{k-1}-s\right)^{\beta-1} y(s) d s \\
& -\lambda \int_{t_{k}}^{t} \frac{(t-s)^{\alpha-1}}{\Gamma(\alpha)} u(s) d s-\lambda \sum_{i=1}^{k} \int_{t_{i-1}}^{t_{i}} \frac{\left(t_{i}-s\right)^{\alpha-1}}{\Gamma(\alpha)} u(s) d s \\
& +\frac{\lambda\left(t-t_{k}\right)^{\alpha}}{\Gamma(\alpha+1)} \sum_{i=1}^{k} I_{i}\left(u\left(t_{i}\right)\right) \\
& +\sum_{i=1}^{k} I_{i}\left(u\left(t_{i}\right)\right)+\sum_{i=2}^{k} \frac{\lambda\left(t_{i}-t_{i-1}\right)^{\alpha}}{\Gamma(\alpha+1)} I_{1}\left(u\left(t_{1}\right)\right) \\
& +\cdots+\sum_{i=k-1}^{k} \frac{\lambda\left(t_{i}-t_{i-1}\right)^{\alpha}}{\Gamma(\alpha+1)} I_{k-2}\left(u\left(t_{k-2}\right)\right) \\
& +\frac{\lambda\left(t_{k}-t_{k-1}\right)^{\alpha}}{\Gamma(\alpha+1)} I_{k-1}\left(u\left(t_{k-1}\right)\right)+\frac{\left(t-t_{k}\right)^{\alpha}}{\Gamma(\alpha+1)} \sum_{i=1}^{k} I_{i}^{* \prime \prime}\left(u\left(t_{i}\right)\right) \\
& +\sum_{i=2}^{k} \frac{\left(t_{i}-t_{i-1}\right)^{\alpha}}{\Gamma(\alpha+1)} I_{1}^{*}\left(u\left(t_{1}\right)\right) \\
& +\cdots+\sum_{i=k-1}^{k} \frac{\left(t_{i}-t_{i-1}\right)^{\alpha}}{\Gamma(\alpha+1)} I_{k-2}^{k}\left(u\left(t_{k-2}\right)\right)+\frac{\left(t_{k}-t_{k-1}\right)^{\alpha}}{\Gamma(\alpha+1)} I_{k-1}^{*}\left(u\left(t_{k-1}\right)\right) \\
& +\frac{C\left(t-t_{k}\right)^{\alpha}}{\Gamma(\alpha+1)}+C \sum_{i=1}^{k} \frac{\left(t_{i}-t_{i-1}\right)^{\alpha}}{\Gamma(\alpha+1)}+\gamma_{1}, \quad t \in J_{k}, k=1,2, \ldots, p, \\
&
\end{aligned}\right.
$$

where

$$
\begin{aligned}
C= & -\left[\sum_{i=1}^{p+1} \frac{\left(t_{i}-t_{i-1}\right)^{\alpha}}{\Gamma(\alpha+1)}\right]^{-1}\left\{\sum_{i=1}^{p+1} \int_{t_{i-1}}^{t_{i}} \frac{\left(t_{i}-s\right)^{\alpha-1}}{\Gamma(\alpha)} \int_{t_{i-1}}^{s} \frac{(s-r)^{\beta-1}}{\Gamma(\beta)} y(r) d r d s\right. \\
& +\frac{\left(1-t_{p}\right)^{\alpha}}{\Gamma(\alpha+1) \Gamma(\beta)} \sum_{i=1}^{p} \int_{t_{i-1}}^{t_{i}}\left(t_{i}-s\right)^{\beta-1} y(s) d s \\
& +\sum_{i=2}^{p} \frac{\left(t_{i}-t_{i-1}\right)^{\alpha}}{\Gamma(\alpha+1) \Gamma(\beta)} \int_{0}^{t_{1}}\left(t_{1}-s\right)^{\beta-1} y(s) d s+\cdots \\
& +\sum_{i=p-1}^{p} \frac{\left(t_{i}-t_{i-1}\right)^{\alpha}}{\Gamma(\alpha+1) \Gamma(\beta)} \int_{t_{p-3}}^{t_{p-2}}\left(t_{p-2}-s\right)^{\beta-1} y(s) d s \\
& +\frac{\left(t_{p}-t_{p-1}\right)^{\alpha}}{\Gamma(\alpha+1) \Gamma(\beta)} \int_{t_{p-2}}^{t_{p-1}}\left(t_{p-1}-s\right)^{\beta-1} y(s) d s \\
& -\lambda \sum_{i=1}^{p+1} \int_{t_{i-1}}^{t_{i}} \frac{\left(t_{i}-s\right)^{\alpha-1}}{\Gamma(\alpha)} u(s) d s+\frac{\left(1-t_{p}\right)^{\alpha}}{\Gamma(\alpha+1)} \sum_{i=1}^{p}\left(\lambda I_{i}+I_{i}^{*}\right)\left(u\left(t_{i}\right)\right) \\
& +\sum_{i=2}^{p} \frac{\lambda\left(t_{i}-t_{i-1}\right)^{\alpha}}{\Gamma(\alpha+1)}\left(\lambda I_{1}+I_{1}^{*}\right)\left(u\left(t_{1}\right)\right) \\
& +\cdots+\sum_{i=p-1}^{p} \frac{\left(t_{i}-t_{i-1}\right)^{\alpha}}{\Gamma(\alpha+1)}\left(\lambda I_{p-2}+I_{p-2}^{*}\right)\left(u\left(t_{p-2}\right)\right) \\
& \left.+\frac{\left(t_{p}-t_{p-1}\right)^{\alpha}}{\Gamma(\alpha+1)}\left(\lambda I_{p-1}+I_{p-1}^{*}\right)\left(u\left(t_{p-1}\right)\right)+\sum_{i=1}^{k} I_{i}\left(u\left(t_{i}\right)\right)+\gamma_{1}-\gamma_{2}\right\} .
\end{aligned}
$$


Proof Let $\left({ }^{C} D^{\alpha}+\lambda\right) u(t)=v(t)$, by (2.2), we have

$$
{ }^{C} D^{\beta} v(t)=y(t)
$$

We may apply Lemma 2.2 to reduce the equation (2.5) to an equivalent integral equation

$$
v(t)=\frac{1}{\Gamma(\beta)} \int_{0}^{t}(t-s)^{\beta-1} y(s) d s-c_{1}, \quad t \in J_{0},
$$

for some $c_{1} \in \mathbb{R}$.

Thus,

$$
{ }^{C} D^{\alpha} u(t)=\frac{1}{\Gamma(\beta)} \int_{0}^{t}(t-s)^{\beta-1} y(s) d s-\lambda u(t)-c_{1}, \quad t \in J_{0},
$$

for some $c_{1} \in \mathbb{R}$.

Similarly, by Lemma 2.2, we have

$$
\begin{aligned}
u(t)= & \int_{0}^{t} \frac{(t-s)^{\alpha-1}}{\Gamma(\alpha)} \int_{0}^{s} \frac{(s-r)^{\beta-1}}{\Gamma(\beta)} y(r) d r d s \\
& -\lambda \int_{0}^{t} \frac{(t-s)^{\alpha-1}}{\Gamma(\alpha)} u(s) d s-\frac{c_{1} t^{\alpha}}{\Gamma(\alpha+1)}-c_{2}, \quad t \in J_{0},
\end{aligned}
$$

for some $c_{1}, c_{2} \in \mathbb{R}$. Combining with $u(0)=\gamma_{1}$, we get that $c_{2}=-\gamma_{1}$.

Substituting the value of $c_{2}$ in (2.7), we have

$$
\begin{aligned}
u(t)= & \int_{0}^{t} \frac{(t-s)^{\alpha-1}}{\Gamma(\alpha)} \int_{0}^{s} \frac{(s-r)^{\beta-1}}{\Gamma(\beta)} y(r) d r d s \\
& -\lambda \int_{0}^{t} \frac{(t-s)^{\alpha-1}}{\Gamma(\alpha)} u(s) d s-\frac{c_{1} t^{\alpha}}{\Gamma(\alpha+1)}+\gamma_{1}, \quad t \in J_{0},
\end{aligned}
$$

for some $c_{1}, c_{2} \in \mathbb{R}$.

If $t \in J_{1}$, then

$$
\begin{aligned}
& v(t)=\frac{1}{\Gamma(\beta)} \int_{t_{1}}^{t}(t-s)^{\beta-1} y(s) d s-d_{1}, \\
& { }^{C} D^{\alpha} u(t)=\frac{1}{\Gamma(\beta)} \int_{t_{1}}^{t}(t-s)^{\beta-1} y(s) d s-\lambda u(t)-d_{1}, \\
& u(t)=\int_{t_{1}}^{t} \frac{(t-s)^{\alpha-1}}{\Gamma(\alpha)} \int_{t_{1}}^{s} \frac{(s-r)^{\beta-1}}{\Gamma(\beta)} y(r) d r d s-\lambda \int_{t_{1}}^{t} \frac{(t-s)^{\alpha-1}}{\Gamma(\alpha)} u(s) d s-\frac{d_{1}\left(t-t_{1}\right)^{\alpha}}{\Gamma(\alpha+1)}-d_{2},
\end{aligned}
$$

for some $d_{1}, d_{2} \in \mathbb{R}$.

Thus, we have

$$
\begin{aligned}
u\left(t_{1}^{-}\right)= & \int_{0}^{t_{1}} \frac{\left(t_{1}-s\right)^{\alpha-1}}{\Gamma(\alpha)} \int_{0}^{s} \frac{(s-r)^{\beta-1}}{\Gamma(\beta)} y(r) d r d s \\
& -\lambda \int_{0}^{t_{1}} \frac{\left(t_{1}-s\right)^{\alpha-1}}{\Gamma(\alpha)} u(s) d s-\frac{c_{1} t_{1}^{\alpha}}{\Gamma(\alpha+1)}+\gamma_{1},
\end{aligned}
$$




$$
\begin{aligned}
& u\left(t_{1}^{+}\right)=-d_{2}, \\
& { }^{C} D^{\alpha} u\left(t_{1}^{-}\right)=\frac{1}{\Gamma(\beta)} \int_{0}^{t_{1}}\left(t_{1}-s\right)^{\beta-1} y(s) d s-\lambda u\left(t_{1}^{-}\right)-c_{1}, \\
& { }^{C} D^{\alpha} u\left(t_{1}^{+}\right)=-\lambda u\left(t_{1}^{+}\right)-d_{1} .
\end{aligned}
$$

In view of $\Delta u\left(t_{1}\right)=u\left(t_{1}^{+}\right)-u\left(t_{1}^{-}\right)=I_{1}\left(u\left(t_{1}\right)\right)$ and $\Delta{ }^{C} D^{\alpha} u\left(t_{1}\right)={ }^{C} D^{\alpha} u\left(t_{1}^{+}\right)-{ }^{C} D^{\alpha} u\left(t_{1}^{-}\right)=$ $I_{1}^{*}\left(u\left(t_{1}\right)\right)$, we have

$$
\begin{aligned}
-d_{1}= & \frac{1}{\Gamma(\beta)} \int_{0}^{t_{1}}\left(t_{1}-s\right)^{\beta-1} y(s) d s+\lambda I_{1}\left(u\left(t_{1}\right)\right)+I_{1}^{*}\left(u\left(t_{1}\right)\right)-c_{1}, \\
-d_{2}= & I_{1}\left(u\left(t_{1}\right)\right)+\int_{0}^{t_{1}} \frac{\left(t_{1}-s\right)^{\alpha-1}}{\Gamma(\alpha)} \int_{0}^{s} \frac{(s-r)^{\beta-1}}{\Gamma(\beta)} y(r) d r d s-\lambda \int_{0}^{t_{1}} \frac{\left(t_{1}-s\right)^{\alpha-1}}{\Gamma(\alpha)} u(s) d s \\
& -\frac{c_{1} t_{1}^{\alpha}}{\Gamma(\alpha+1)}+\gamma_{1} .
\end{aligned}
$$

Hence,

$$
\begin{aligned}
u(t)= & \int_{t_{1}}^{t} \frac{(t-s)^{\alpha-1}}{\Gamma(\alpha)} \int_{t_{1}}^{s} \frac{(s-r)^{\beta-1}}{\Gamma(\beta)} y(r) d r d s+\int_{0}^{t_{1}} \frac{\left(t_{1}-s\right)^{\alpha-1}}{\Gamma(\alpha)} \int_{0}^{s} \frac{(s-r)^{\beta-1}}{\Gamma(\beta)} y(r) d r d s \\
& +\frac{\left(t-t_{1}\right)^{\alpha}}{\Gamma(\alpha+1) \Gamma(\beta)} \int_{0}^{t_{1}}\left(t_{1}-s\right)^{\beta-1} y(s) d s-\lambda \int_{t_{1}}^{t} \frac{(t-s)^{\alpha-1}}{\Gamma(\alpha)} u(s) d s \\
& -\lambda \int_{0}^{t_{1}} \frac{\left(t_{1}-s\right)^{\alpha-1}}{\Gamma(\alpha)} u(s) d s+\frac{\lambda\left(t-t_{1}\right)^{\alpha}}{\Gamma(\alpha+1)} I_{1}\left(u\left(t_{1}\right)\right)+I_{1}\left(u\left(t_{1}\right)\right) \\
& +\frac{\left(t-t_{1}\right)^{\alpha}}{\Gamma(\alpha+1)} I_{1}^{\prime \prime}\left(u\left(t_{1}\right)\right)-\frac{c_{1}\left(t-t_{1}\right)^{\alpha}}{\Gamma(\alpha+1)}-\frac{c_{1} t_{1}^{\alpha}}{\Gamma(\alpha+1)}+\gamma_{1}, \quad t \in J_{1} .
\end{aligned}
$$

By a similar process, we can get

$$
\begin{aligned}
u(t)= & \int_{t_{2}}^{t} \frac{(t-s)^{\alpha-1}}{\Gamma(\alpha)} \int_{t_{2}}^{s} \frac{(s-r)^{\beta-1}}{\Gamma(\beta)} y(r) d r d s+\int_{t_{1}}^{t_{2}} \frac{\left(t_{2}-s\right)^{\alpha-1}}{\Gamma(\alpha)} \int_{t_{1}}^{s} \frac{(s-r)^{\beta-1}}{\Gamma(\beta)} y(r) d r d s \\
& +\int_{0}^{t_{1}} \frac{\left(t_{1}-s\right)^{\alpha-1}}{\Gamma(\alpha)} \int_{0}^{s} \frac{(s-r)^{\beta-1}}{\Gamma(\beta)} y(r) d r d s+\frac{\left(t_{2}-t_{1}\right)^{\alpha}}{\Gamma(\alpha+1) \Gamma(\beta)} \int_{0}^{t_{1}}\left(t_{1}-s\right)^{\beta-1} y(s) d s \\
& +\frac{\left(t-t_{2}\right)^{\alpha}}{\Gamma(\alpha+1) \Gamma(\beta)} \int_{t_{1}}^{t_{2}}\left(t_{2}-s\right)^{\beta-1} y(s) d s+\frac{\left(t-t_{2}\right)^{\alpha}}{\Gamma(\alpha+1) \Gamma(\beta)} \int_{0}^{t_{1}}\left(t_{1}-s\right)^{\beta-1} y(s) d s \\
& -\lambda \int_{t_{2}}^{t} \frac{(t-s)^{\alpha-1}}{\Gamma(\alpha)} u(s) d s-\lambda \int_{t_{1}}^{t_{2}} \frac{\left(t_{2}-s\right)^{\alpha-1}}{\Gamma(\alpha)} u(s) d s-\lambda \int_{0}^{t_{1}} \frac{\left(t_{1}-s\right)^{\alpha-1}}{\Gamma(\alpha)} u(s) d s \\
& +\frac{\lambda\left(t-t_{2}\right)^{\alpha}}{\Gamma(\alpha+1)} I_{2}\left(u\left(t_{2}\right)\right)+\frac{\lambda\left(t-t_{2}\right)^{\alpha}}{\Gamma(\alpha+1)} I_{1}\left(u\left(t_{1}\right)\right)+\frac{\lambda\left(t_{2}-t_{1}\right)^{\alpha}}{\Gamma(\alpha+1)} I_{1}\left(u\left(t_{1}\right)\right) \\
& +I_{2}\left(u\left(t_{2}\right)\right)+I_{1}\left(u\left(t_{1}\right)\right)+\frac{\left(t-t_{2}\right)^{\alpha}}{\Gamma(\alpha+1)} I_{2}^{*}\left(u\left(t_{2}\right)\right)+\frac{\left(t-t_{2}\right)^{\alpha}}{\Gamma(\alpha+1)} I_{1}^{*}\left(u\left(t_{1}\right)\right) \\
& +\frac{\left(t_{2}-t_{1}\right)^{\alpha}}{\Gamma(\alpha+1)} I_{1}^{*}\left(u\left(t_{1}\right)\right)-\frac{c_{1}\left(t-t_{2}\right)^{\alpha}}{\Gamma(\alpha+1)} \\
& -\frac{c_{1}\left(t_{2}-t_{1}\right)^{\alpha}}{\Gamma(\alpha+1)}-\frac{c_{1} t_{1}^{\alpha}}{\Gamma(\alpha+1)}+\gamma_{1}, \quad t \in J_{2}
\end{aligned}
$$


and

$$
\begin{aligned}
& u(t)=\int_{t_{3}}^{t} \frac{(t-s)^{\alpha-1}}{\Gamma(\alpha)} \int_{t_{3}}^{s} \frac{(s-r)^{\beta-1}}{\Gamma(\beta)} y(r) d r d s+\int_{t_{2}}^{t_{3}} \frac{\left(t_{3}-s\right)^{\alpha-1}}{\Gamma(\alpha)} \int_{t_{2}}^{s} \frac{(s-r)^{\beta-1}}{\Gamma(\beta)} y(r) d r d s \\
& +\int_{t_{1}}^{t_{2}} \frac{\left(t_{2}-s\right)^{\alpha-1}}{\Gamma(\alpha)} \int_{t_{1}}^{s} \frac{(s-r)^{\beta-1}}{\Gamma(\beta)} y(r) d r d s+\int_{0}^{t_{1}} \frac{\left(t_{1}-s\right)^{\alpha-1}}{\Gamma(\alpha)} \int_{0}^{s} \frac{(s-r)^{\beta-1}}{\Gamma(\beta)} y(r) d r d s \\
& +\frac{\left(t-t_{3}\right)^{\alpha}}{\Gamma(\alpha+1) \Gamma(\beta)} \int_{t_{2}}^{t_{3}}\left(t_{3}-s\right)^{\beta-1} y(s) d s+\frac{\left(t-t_{3}\right)^{\alpha}}{\Gamma(\alpha+1) \Gamma(\beta)} \int_{t_{1}}^{t_{2}}\left(t_{2}-s\right)^{\beta-1} y(s) d s \\
& +\frac{\left(t-t_{3}\right)^{\alpha}}{\Gamma(\alpha+1) \Gamma(\beta)} \int_{0}^{t_{1}}\left(t_{1}-s\right)^{\beta-1} y(s) d s+\frac{\left(t_{2}-t_{1}\right)^{\alpha}}{\Gamma(\alpha+1) \Gamma(\beta)} \int_{0}^{t_{1}}\left(t_{1}-s\right)^{\beta-1} y(s) d s \\
& +\frac{\left(t_{3}-t_{2}\right)^{\alpha}}{\Gamma(\alpha+1) \Gamma(\beta)} \int_{0}^{t_{1}}\left(t_{1}-s\right)^{\beta-1} y(s) d s+\frac{\left(t_{3}-t_{2}\right)^{\alpha}}{\Gamma(\alpha+1) \Gamma(\beta)} \int_{t_{1}}^{t_{2}}\left(t_{2}-s\right)^{\beta-1} y(s) d s \\
& -\lambda \int_{t_{3}}^{t} \frac{(t-s)^{\alpha-1}}{\Gamma(\alpha)} u(s) d s-\lambda \int_{t_{2}}^{t_{3}} \frac{\left(t_{3}-s\right)^{\alpha-1}}{\Gamma(\alpha)} u(s) d s-\lambda \int_{t_{1}}^{t_{2}} \frac{\left(t_{2}-s\right)^{\alpha-1}}{\Gamma(\alpha)} u(s) d s \\
& -\lambda \int_{0}^{t_{1}} \frac{\left(t_{1}-s\right)^{\alpha-1}}{\Gamma(\alpha)} u(s) d s+\frac{\lambda\left(t-t_{3}\right)^{\alpha}}{\Gamma(\alpha+1)} I_{3}\left(u\left(t_{3}\right)\right)+\frac{\lambda\left(t-t_{3}\right)^{\alpha}}{\Gamma(\alpha+1)} I_{2}\left(u\left(t_{2}\right)\right) \\
& +\frac{\lambda\left(t-t_{3}\right)^{\alpha}}{\Gamma(\alpha+1)} I_{1}\left(u\left(t_{1}\right)\right)+\frac{\lambda\left(t_{3}-t_{2}\right)^{\alpha}}{\Gamma(\alpha+1)} I_{2}\left(u\left(t_{2}\right)\right)+\frac{\lambda\left(t_{3}-t_{2}\right)^{\alpha}}{\Gamma(\alpha+1)} I_{1}\left(u\left(t_{1}\right)\right) \\
& +\frac{\lambda\left(t_{2}-t_{1}\right)^{\alpha}}{\Gamma(\alpha+1)} I_{1}\left(u\left(t_{1}\right)\right)+I_{3}\left(u\left(t_{3}\right)\right)+I_{2}\left(u\left(t_{2}\right)\right)+I_{1}\left(u\left(t_{1}\right)\right) \\
& +\frac{\left(t-t_{3}\right)^{\alpha}}{\Gamma(\alpha+1)} I_{3}^{*}\left(u\left(t_{3}\right)\right)+\frac{\left(t-t_{3}\right)^{\alpha}}{\Gamma(\alpha+1)} I_{2}^{*}\left(u\left(t_{2}\right)\right)+\frac{\left(t-t_{3}\right)^{\alpha}}{\Gamma(\alpha+1)} I_{1}^{*}\left(u\left(t_{1}\right)\right) \\
& +\frac{\left(t_{3}-t_{2}\right)^{\alpha}}{\Gamma(\alpha+1)} I_{2}^{*}\left(u\left(t_{2}\right)\right)+\frac{\left(t_{3}-t_{2}\right)^{\alpha}}{\Gamma(\alpha+1)} I_{1}^{*}\left(u\left(t_{1}\right)\right)+\frac{\left(t_{2}-t_{1}\right)^{\alpha}}{\Gamma(\alpha+1)} I_{1}^{*}\left(u\left(t_{1}\right)\right) \\
& -\frac{c_{1}\left(t-t_{3}\right)^{\alpha}}{\Gamma(\alpha+1)}-\frac{c_{1}\left(t_{3}-t_{2}\right)^{\alpha}}{\Gamma(\alpha+1)}-\frac{c_{1}\left(t_{2}-t_{1}\right)^{\alpha}}{\Gamma(\alpha+1)}-\frac{c_{1} t_{1}^{\alpha}}{\Gamma(\alpha+1)}+\gamma_{1}, \quad t \in J_{3} .
\end{aligned}
$$

By the same method, for $t \in J_{k}$, we have

$$
\begin{aligned}
u(t)= & \int_{t_{k}}^{t} \frac{(t-s)^{\alpha-1}}{\Gamma(\alpha)} \int_{t_{k}}^{s} \frac{(s-r)^{\beta-1}}{\Gamma(\beta)} y(r) d r d s \\
& +\sum_{i=1}^{k} \int_{t_{i-1}}^{t_{i}} \frac{\left(t_{i}-s\right)^{\alpha-1}}{\Gamma(\alpha)} \int_{t_{i-1}}^{s} \frac{(s-r)^{\beta-1}}{\Gamma(\beta)} y(r) d r d s \\
& +\frac{\left(t-t_{k}\right)^{\alpha}}{\Gamma(\alpha+1) \Gamma(\beta)} \sum_{i=1}^{k} \int_{t_{i-1}}^{t_{i}}\left(t_{i}-s\right)^{\beta-1} y(s) d s \\
& +\sum_{i=2}^{k} \frac{\left(t_{i}-t_{i-1}\right)^{\alpha}}{\Gamma(\alpha+1) \Gamma(\beta)} \int_{0}^{t_{1}}\left(t_{1}-s\right)^{\beta-1} y(s) d s \\
& +\cdots+\sum_{i=k-1}^{k} \frac{\left(t_{i}-t_{i-1}\right)^{\alpha}}{\Gamma(\alpha+1) \Gamma(\beta)} \int_{t_{k-3}}^{t_{k-2}}\left(t_{k-2}-s\right)^{\beta-1} y(s) d s \\
& +\frac{\left(t_{k}-t_{k-1}\right)^{\alpha}}{\Gamma(\alpha+1) \Gamma(\beta)} \int_{t_{k-2}}^{t_{k-1}}\left(t_{k-1}-s\right)^{\beta-1} y(s) d s-\lambda \int_{t_{k}}^{t} \frac{(t-s)^{\alpha-1}}{\Gamma(\alpha)} u(s) d s
\end{aligned}
$$




$$
\begin{aligned}
& -\lambda \sum_{i=1}^{k} \int_{t_{i-1}}^{t_{i}} \frac{\left(t_{i}-s\right)^{\alpha-1}}{\Gamma(\alpha)} u(s) d s+\frac{\lambda\left(t-t_{k}\right)^{\alpha}}{\Gamma(\alpha+1)} \sum_{i=1}^{k} I_{i}\left(u\left(t_{i}\right)\right)+\sum_{i=1}^{k} I_{i}\left(u\left(t_{i}\right)\right) \\
& +\sum_{i=2}^{k} \frac{\lambda\left(t_{i}-t_{i-1}\right)^{\alpha}}{\Gamma(\alpha+1)} I_{1}\left(u\left(t_{1}\right)\right)+\cdots+\sum_{i=k-1}^{k} \frac{\lambda\left(t_{i}-t_{i-1}\right)^{\alpha}}{\Gamma(\alpha+1)} I_{k-2}\left(u\left(t_{k-2}\right)\right) \\
& +\frac{\lambda\left(t_{k}-t_{k-1}\right)^{\alpha}}{\Gamma(\alpha+1)} I_{k-1}\left(u\left(t_{k-1}\right)\right)+\frac{\left(t-t_{k}\right)^{\alpha}}{\Gamma(\alpha+1)} \sum_{i=1}^{k} I_{i}^{* \prime}\left(u\left(t_{i}\right)\right)+\sum_{i=2}^{k} \frac{\left(t_{i}-t_{i-1}\right)^{\alpha}}{\Gamma(\alpha+1)} I_{1}^{*}\left(u\left(t_{1}\right)\right) \\
& +\cdots+\sum_{i=k-1}^{k} \frac{\left(t_{i}-t_{i-1}\right)^{\alpha}}{\Gamma(\alpha+1)} I_{k-2}^{*}\left(u\left(t_{k-2}\right)\right)+\frac{\left(t_{k}-t_{k-1}\right)^{\alpha}}{\Gamma(\alpha+1)} I_{k-1}^{*}\left(u\left(t_{k-1}\right)\right) \\
& -\frac{c_{1}\left(t-t_{k}\right)^{\alpha}}{\Gamma(\alpha+1)}-c_{1} \sum_{i=1}^{k} \frac{\left(t_{i}-t_{i-1}\right)^{\alpha}}{\Gamma(\alpha+1)}+\gamma_{1} .
\end{aligned}
$$

By (2.12) and the condition $u(1)=\gamma_{2}$, we have

$$
\begin{aligned}
c_{1}= & {\left[\sum_{i=1}^{p+1} \frac{\left(t_{i}-t_{i-1}\right)^{\alpha}}{\Gamma(\alpha+1)}\right]^{-1}\left\{\sum_{i=1}^{p+1} \int_{t_{i-1}}^{t_{i}} \frac{\left(t_{i}-s\right)^{\alpha-1}}{\Gamma(\alpha)} \int_{t_{i-1}}^{s} \frac{(s-r)^{\beta-1}}{\Gamma(\beta)} y(r) d r d s\right.} \\
& +\frac{\left(1-t_{p}\right)^{\alpha}}{\Gamma(\alpha+1) \Gamma(\beta)} \sum_{i=1}^{p} \int_{t_{i-1}}^{t_{i}}\left(t_{i}-s\right)^{\beta-1} y(s) d s+\sum_{i=2}^{p} \frac{\left(t_{i}-t_{i-1}\right)^{\alpha}}{\Gamma(\alpha+1) \Gamma(\beta)} \int_{0}^{t_{1}}\left(t_{1}-s\right)^{\beta-1} y(s) d s \\
& +\cdots+\sum_{i=p-1}^{p} \frac{\left(t_{i}-t_{i-1}\right)^{\alpha}}{\Gamma(\alpha+1) \Gamma(\beta)} \int_{t_{p-3}}^{t_{p-2}}\left(t_{p-2}-s\right)^{\beta-1} y(s) d s \\
& +\frac{\left(t_{p}-t_{p-1}\right)^{\alpha}}{\Gamma(\alpha+1) \Gamma(\beta)} \int_{t_{p-2}}^{t_{p-1}}\left(t_{p-1}-s\right)^{\beta-1} y(s) d s-\lambda \sum_{i=1}^{p+1} \int_{t_{i-1}}^{t_{i}} \frac{\left(t_{i}-s\right)^{\alpha-1}}{\Gamma(\alpha)} u(s) d s \\
& +\frac{\left(1-t_{p}\right)^{\alpha}}{\Gamma(\alpha+1)} \sum_{i=1}^{p}\left(\lambda I_{i}+I_{i}^{*}\right)\left(u\left(t_{i}\right)\right)+\sum_{i=2}^{p} \frac{\lambda\left(t_{i}-t_{i-1}\right)^{\alpha}}{\Gamma(\alpha+1)}\left(\lambda I_{1}+I_{1}^{*}\right)\left(u\left(t_{1}\right)\right)+\cdots \\
& +\sum_{i=p-1}^{p} \frac{\left(t_{i}-t_{i-1}\right)^{\alpha}}{\Gamma(\alpha+1)}\left(\lambda I_{p-2}+I_{p-2}^{*}\right)\left(u\left(t_{p-2}\right)\right)+\frac{\left(t_{p}-t_{p-1}\right)^{\alpha}}{\Gamma(\alpha+1)}\left(\lambda I_{p-1}+I_{p-1}^{*}\right)\left(u\left(t_{p-1}\right)\right) \\
& \left.+\sum_{i=1}^{k} I_{i}\left(u\left(t_{i}\right)\right)+\gamma_{1}-\gamma_{2}\right\} .
\end{aligned}
$$

Substituting the value of $c_{1}$ in (2.8) and (2.12) and letting $C=-c_{1}$, we can get (2.3). Conversely, assume that $u$ is a solution of the impulsive fractional integral equation (2.3). Then by a direct computation, it follows that the solution given by (2.3) satisfies (2.2). This completes the proof.

\subsection{Nonlinear problem}

Define the constant:

$$
\begin{aligned}
\Lambda= & \frac{2(p+1) L_{1}}{\Gamma(\alpha+1+\beta)}+\frac{p(p+1) L_{1}}{\Gamma(\alpha+1) \Gamma(\beta+1)} \\
& +\frac{2(p+1)|\lambda|}{\Gamma(\alpha+1)}+\frac{p(p+1)\left(|\lambda| L_{2}+L_{3}\right)}{\Gamma(\alpha+1)}+2 p L_{2} .
\end{aligned}
$$


Theorem 2.1 Assume that

$\left(\mathrm{H}_{1}\right)$ There exist constants $L_{i}(i=1,2,3)$ such that

$$
\begin{aligned}
& \|f(t, u)-f(t, v)\| \leq L_{1}\|u-v\|, \quad\left\|I_{k}(u)-I_{k}(v)\right\| \leq L_{2}\|u-v\|, \\
& \left\|I_{k}^{*}(u)-I_{k}^{*}(v)\right\| \leq L_{3}\|u-v\|, \quad \text { for } t \in J, u, v \in E \text { and } k=1,2, \ldots, p .
\end{aligned}
$$

Then problem (2.1) has a unique solution provided $\Lambda<1$, where $\Lambda$ is given by (2.14).

Proof Define the operator $T: P C(J, E) \rightarrow P C(J, E)$ as follows:

$$
\begin{aligned}
& \operatorname{Tu}(t)=\int_{t_{k}}^{t} \frac{(t-s)^{\alpha-1}}{\Gamma(\alpha)} \int_{t_{k}}^{s} \frac{(s-r)^{\beta-1}}{\Gamma(\beta)} f(r, u(r)) d r d s \\
& +\sum_{i=1}^{k} \int_{t_{i-1}}^{t_{i}} \frac{\left(t_{i}-s\right)^{\alpha-1}}{\Gamma(\alpha)} \int_{t_{i-1}}^{s} \frac{(s-r)^{\beta-1}}{\Gamma(\beta)} f(r, u(r)) d r d s \\
& +\frac{\left(t-t_{k}\right)^{\alpha}}{\Gamma(\alpha+1) \Gamma(\beta)} \sum_{i=1}^{k} \int_{t_{i-1}}^{t_{i}}\left(t_{i}-s\right)^{\beta-1} f(s, u(s)) d s \\
& +\sum_{i=2}^{k} \frac{\left(t_{i}-t_{i-1}\right)^{\alpha}}{\Gamma(\alpha+1) \Gamma(\beta)} \int_{0}^{t_{1}}\left(t_{1}-s\right)^{\beta-1} f(s, u(s)) d s+\cdots \\
& +\sum_{i=k-1}^{k} \frac{\left(t_{i}-t_{i-1}\right)^{\alpha}}{\Gamma(\alpha+1) \Gamma(\beta)} \int_{t_{k-3}}^{t_{k-2}}\left(t_{k-2}-s\right)^{\beta-1} f(s, u(s)) d s \\
& +\frac{\left(t_{k}-t_{k-1}\right)^{\alpha}}{\Gamma(\alpha+1) \Gamma(\beta)} \int_{t_{k-2}}^{t_{k-1}}\left(t_{k-1}-s\right)^{\beta-1} f(s, u(s)) d s \\
& -\lambda \int_{t_{k}}^{t} \frac{(t-s)^{\alpha-1}}{\Gamma(\alpha)} u(s) d s-\lambda \sum_{i=1}^{k} \int_{t_{i-1}}^{t_{i}} \frac{\left(t_{i}-s\right)^{\alpha-1}}{\Gamma(\alpha)} u(s) d s \\
& +\frac{\left(t-t_{k}\right)^{\alpha}}{\Gamma(\alpha+1)} \sum_{i=1}^{k}\left(\lambda I_{i}+I_{i}^{*}\right)\left(u\left(t_{i}\right)\right)+\sum_{i=2}^{k} \frac{\left(t_{i}-t_{i-1}\right)^{\alpha}}{\Gamma(\alpha+1)}\left(\lambda I_{1}+I_{1}^{*}\right)\left(u\left(t_{1}\right)\right)+\cdots \\
& +\sum_{i=k-1}^{k} \frac{\left(t_{i}-t_{i-1}\right)^{\alpha}}{\Gamma(\alpha+1)}\left(\lambda I_{k-2}+I_{k-2}^{*}\right)\left(u\left(t_{k-2}\right)\right) \\
& +\frac{\left(t_{k}-t_{k-1}\right)^{\alpha}}{\Gamma(\alpha+1)}\left(\lambda I_{k-1}+I_{k-1}^{*}\right)\left(u\left(t_{k-1}\right)\right)+\sum_{i=1}^{k} I_{i}\left(u\left(t_{i}\right)\right) \\
& +\frac{C\left(t-t_{k}\right)^{\alpha}}{\Gamma(\alpha+1)}+C \sum_{i=1}^{k} \frac{\left(t_{i}-t_{i-1}\right)^{\alpha}}{\Gamma(\alpha+1)}+\gamma_{1}
\end{aligned}
$$

where

$$
\begin{aligned}
C= & -\left[\sum_{i=1}^{p+1} \frac{\left(t_{i}-t_{i-1}\right)^{\alpha}}{\Gamma(\alpha+1)}\right]^{-1}\left\{\sum_{i=1}^{p+1} \int_{t_{i-1}}^{t_{i}} \frac{\left(t_{i}-s\right)^{\alpha-1}}{\Gamma(\alpha)} \int_{t_{i-1}}^{s} \frac{(s-r)^{\beta-1}}{\Gamma(\beta)} f(r, u(r)) d r d s\right. \\
& +\frac{\left(1-t_{p}\right)^{\alpha}}{\Gamma(\alpha+1) \Gamma(\beta)} \sum_{i=1}^{p} \int_{t_{i-1}}^{t_{i}}\left(t_{i}-s\right)^{\beta-1} f(s, u(s)) d s
\end{aligned}
$$




$$
\begin{aligned}
& +\sum_{i=2}^{p} \frac{\left(t_{i}-t_{i-1}\right)^{\alpha}}{\Gamma(\alpha+1) \Gamma(\beta)} \int_{0}^{t_{1}}\left(t_{1}-s\right)^{\beta-1} f(s, u(s)) d s \\
& +\cdots+\sum_{i=p-1}^{p} \frac{\left(t_{i}-t_{i-1}\right)^{\alpha}}{\Gamma(\alpha+1) \Gamma(\beta)} \int_{t_{p-3}}^{t_{p-2}}\left(t_{p-2}-s\right)^{\beta-1} f(s, u(s)) d s \\
& +\frac{\left(t_{p}-t_{p-1}\right)^{\alpha}}{\Gamma(\alpha+1) \Gamma(\beta)} \int_{t_{p-2}}^{t_{p-1}}\left(t_{p-1}-s\right)^{\beta-1} f(s, u(s)) d s-\lambda \sum_{i=1}^{p+1} \int_{t_{i-1}}^{t_{i}} \frac{\left(t_{i}-s\right)^{\alpha-1}}{\Gamma(\alpha)} u(s) d s \\
& +\frac{\left(1-t_{p}\right)^{\alpha}}{\Gamma(\alpha+1)} \sum_{i=1}^{p}\left(\lambda I_{i}+I_{i}^{*}\right)\left(u\left(t_{i}\right)\right)+\sum_{i=2}^{p} \frac{\lambda\left(t_{i}-t_{i-1}\right)^{\alpha}}{\Gamma(\alpha+1)}\left(\lambda I_{1}+I_{1}^{*}\right)\left(u\left(t_{1}\right)\right)+\cdots \\
& +\sum_{i=p-1}^{p} \frac{\left(t_{i}-t_{i-1}\right)^{\alpha}}{\Gamma(\alpha+1)}\left(\lambda I_{p-2}+I_{p-2}^{*}\right)\left(u\left(t_{p-2}\right)\right)+\frac{\left(t_{p}-t_{p-1}\right)^{\alpha}}{\Gamma(\alpha+1)}\left(\lambda I_{p-1}+I_{p-1}^{*}\right)\left(u\left(t_{p-1}\right)\right) \\
& \left.+\sum_{i=1}^{k} I_{i}\left(u\left(t_{i}\right)\right)+\gamma_{1}-\gamma_{2}\right\} .
\end{aligned}
$$

Then the equation (2.1) has a solution if and only if the operator $T$ has a fixed point. Let $u, v \in P C(J, E)$. By (2.15), we have

$$
\begin{aligned}
& \|(T u)(t)-(T v)(t)\| \\
& \leq \int_{t_{k}}^{t} \frac{(t-s)^{\alpha-1}}{\Gamma(\alpha)} \int_{t_{k}}^{s} \frac{(s-r)^{\beta-1}}{\Gamma(\beta)}\|f(r, u(r))-f(r, v(r))\| d r d s \\
& +\sum_{i=1}^{k} \int_{t_{i-1}}^{t_{i}} \frac{\left(t_{i}-s\right)^{\alpha-1}}{\Gamma(\alpha)} \int_{t_{i-1}}^{s} \frac{(s-r)^{\beta-1}}{\Gamma(\beta)}\|f(r, u(r))-f(r, v(r))\| d r d s \\
& +\frac{\left(t-t_{k}\right)^{\alpha}}{\Gamma(\alpha+1) \Gamma(\beta)} \sum_{i=1}^{k} \int_{t_{i-1}}^{t_{i}}\left(t_{i}-s\right)^{\beta-1}\|f(s, u(s))-f(s, v(s))\| d s \\
& +\sum_{i=2}^{k} \frac{\left(t_{i}-t_{i-1}\right)^{\alpha}}{\Gamma(\alpha+1) \Gamma(\beta)} \int_{0}^{t_{1}}\left(t_{1}-s\right)^{\beta-1}\|f(s, u(s))-f(s, v(s))\| d s+\cdots \\
& +\sum_{i=k-1}^{k} \frac{\left(t_{i}-t_{i-1}\right)^{\alpha}}{\Gamma(\alpha+1) \Gamma(\beta)} \int_{t_{k-3}}^{t_{k-2}}\left(t_{k-2}-s\right)^{\beta-1}\|f(s, u(s))-f(s, v(s))\| d s \\
& +\frac{\left(t_{k}-t_{k-1}\right)^{\alpha}}{\Gamma(\alpha+1) \Gamma(\beta)} \int_{t_{k-2}}^{t_{k-1}}\left(t_{k-1}-s\right)^{\beta-1}\|f(s, u(s))-f(s, v(s))\| d s \\
& +|\lambda| \int_{t_{k}}^{t} \frac{(t-s)^{\alpha-1}}{\Gamma(\alpha)}\|u(s)-v(s)\| d s \\
& +|\lambda| \sum_{i=1}^{k} \int_{t_{i-1}}^{t_{i}} \frac{\left(t_{i}-s\right)^{\alpha-1}}{\Gamma(\alpha)}\|u(s)-v(s)\| d s \\
& +\frac{\left(t-t_{k}\right)^{\alpha}}{\Gamma(\alpha+1)} \sum_{i=1}^{k}\left\|\left(\lambda I_{i}+I_{i}^{*}\right)\left(u\left(t_{i}\right)\right)-\left(\lambda I_{i}+I_{i}^{\prime \prime}\right)\left(v\left(t_{i}\right)\right)\right\| \\
& +\sum_{i=2}^{k} \frac{\left(t_{i}-t_{i-1}\right)^{\alpha}}{\Gamma(\alpha+1)}\left\|\left(\lambda I_{1}+I_{1}^{*}\right)\left(u\left(t_{1}\right)\right)-\left(\lambda I_{1}+I_{1}^{*}\right)\left(v\left(t_{1}\right)\right)\right\|+\cdots
\end{aligned}
$$




$$
\begin{aligned}
& +\sum_{i=k-1}^{k} \frac{\left(t_{i}-t_{i-1}\right)^{\alpha}}{\Gamma(\alpha+1)}\left\|\left(\lambda I_{k-2}+I_{k-2}^{*}\right)\left(u\left(t_{k-2}\right)\right)-\left(\lambda I_{k-2}+I_{k-2}^{*}\right)\left(v\left(t_{k-2}\right)\right)\right\| \\
& +\frac{\left(t_{k}-t_{k-1}\right)^{\alpha}}{\Gamma(\alpha+1)}\left\|\left(\lambda I_{k-1}+I_{k-1}^{*}\right)\left(u\left(t_{k-1}\right)\right)-\left(\lambda I_{k-1}+I_{k-1}^{*}\right)\left(v\left(t_{k-1}\right)\right)\right\| \\
& +\sum_{i=1}^{k}\left\|I_{i}\left(u\left(t_{i}\right)\right)-I_{i}\left(v\left(t_{i}\right)\right)\right\| \\
& +\int_{t_{k}}^{t} \frac{(t-s)^{\alpha-1}}{\Gamma(\alpha)} \int_{t_{k}}^{s} \frac{(s-r)^{\beta-1}}{\Gamma(\beta)}\|f(r, u(r))-f(r, v(r))\| d r d s \\
& +\sum_{i=1}^{k} \int_{t_{i-1}}^{t_{i}} \frac{\left(t_{i}-s\right)^{\alpha-1}}{\Gamma(\alpha)} \int_{t_{i-1}}^{s} \frac{(s-r)^{\beta-1}}{\Gamma(\beta)}\|f(r, u(r))-f(r, v(r))\| d r d s \\
& +\frac{\left(t-t_{k}\right)^{\alpha}}{\Gamma(\alpha+1) \Gamma(\beta)} \sum_{i=1}^{k} \int_{t_{i-1}}^{t_{i}}\left(t_{i}-s\right)^{\beta-1}\|f(s, u(s))-f(s, v(s))\| d s \\
& +\sum_{i=2}^{k} \frac{\left(t_{i}-t_{i-1}\right)^{\alpha}}{\Gamma(\alpha+1) \Gamma(\beta)} \int_{0}^{t_{1}}\left(t_{1}-s\right)^{\beta-1}\|f(s, u(s))-f(s, v(s))\| d s+\cdots \\
& +\sum_{i=k-1}^{k} \frac{\left(t_{i}-t_{i-1}\right)^{\alpha}}{\Gamma(\alpha+1) \Gamma(\beta)} \int_{t_{k-3}}^{t_{k-2}}\left(t_{k-2}-s\right)^{\beta-1}\|f(s, u(s))-f(s, v(s))\| d s \\
& +\frac{\left(t_{k}-t_{k-1}\right)^{\alpha}}{\Gamma(\alpha+1) \Gamma(\beta)} \int_{t_{k-2}}^{t_{k-1}}\left(t_{k-1}-s\right)^{\beta-1}\|f(s, u(s))-f(s, v(s))\| d s \\
& +|\lambda| \int_{t_{k}}^{t} \frac{(t-s)^{\alpha-1}}{\Gamma(\alpha)}\|u(s)-v(s)\| d s+|\lambda| \sum_{i=1}^{k} \int_{t_{i-1}}^{t_{i}} \frac{\left(t_{i}-s\right)^{\alpha-1}}{\Gamma(\alpha)}\|u(s)-v(s)\| d s \\
& +\frac{\left(t-t_{k}\right)^{\alpha}}{\Gamma(\alpha+1)} \sum_{i=1}^{k}\left\|\left(\lambda I_{i}+I_{i}^{*}\right)\left(u\left(t_{i}\right)\right)-\left(\lambda I_{i}+I_{i}^{*}\right)\left(v\left(t_{i}\right)\right)\right\| \\
& +\sum_{i=2}^{k} \frac{\left(t_{i}-t_{i-1}\right)^{\alpha}}{\Gamma(\alpha+1)}\left\|\left(\lambda I_{1}+I_{1}^{*}\right)\left(u\left(t_{1}\right)\right)-\left(\lambda I_{1}+I_{1}^{*}\right)\left(v\left(t_{1}\right)\right)\right\|+\cdots \\
& +\sum_{i=k-1}^{k} \frac{\left(t_{i}-t_{i-1}\right)^{\alpha}}{\Gamma(\alpha+1)}\left\|\left(\lambda I_{k-2}+I_{k-2}^{*}\right)\left(u\left(t_{k-2}\right)\right)-\left(\lambda I_{k-2}+I_{k-2}^{*}\right)\left(v\left(t_{k-2}\right)\right)\right\| \\
& +\frac{\left(t_{k}-t_{k-1}\right)^{\alpha}}{\Gamma(\alpha+1)}\left\|\left(\lambda I_{k-1}+I_{k-1}^{*}\right)\left(u\left(t_{k-1}\right)\right)-\left(\lambda I_{k-1}+I_{k-1}^{*}\right)\left(v\left(t_{k-1}\right)\right)\right\| \\
& +\sum_{i=1}^{k}\left\|I_{i}\left(u\left(t_{i}\right)\right)-I_{i}\left(v\left(t_{i}\right)\right)\right\| \\
& \leq 2\left\{\int_{t_{k}}^{t} \frac{(t-s)^{\alpha-1}}{\Gamma(\alpha)} \int_{t_{k}}^{s} \frac{(s-r)^{\beta-1}}{\Gamma(\beta)}\|f(r, u(r))-f(r, v(r))\| d r d s\right. \\
& +\sum_{i=1}^{k} \int_{t_{i-1}}^{t_{i}} \frac{\left(t_{i}-s\right)^{\alpha-1}}{\Gamma(\alpha)} \int_{t_{i-1}}^{s} \frac{(s-r)^{\beta-1}}{\Gamma(\beta)}\|f(r, u(r))-f(r, v(r))\| d r d s \\
& +\frac{\left(t-t_{k}\right)^{\alpha}}{\Gamma(\alpha+1) \Gamma(\beta)} \sum_{i=1}^{k} \int_{t_{i-1}}^{t_{i}}\left(t_{i}-s\right)^{\beta-1}\|f(s, u(s))-f(s, v(s))\| d s
\end{aligned}
$$




$$
\begin{aligned}
& +\sum_{i=2}^{k} \frac{\left(t_{i}-t_{i-1}\right)^{\alpha}}{\Gamma(\alpha+1) \Gamma(\beta)} \int_{0}^{t_{1}}\left(t_{1}-s\right)^{\beta-1}\|f(s, u(s))-f(s, v(s))\| d s+\cdots \\
& +\sum_{i=k-1}^{k} \frac{\left(t_{i}-t_{i-1}\right)^{\alpha}}{\Gamma(\alpha+1) \Gamma(\beta)} \int_{t_{k-3}}^{t_{k-2}}\left(t_{k-2}-s\right)^{\beta-1}\|f(s, u(s))-f(s, v(s))\| d s \\
& +\frac{\left(t_{k}-t_{k-1}\right)^{\alpha}}{\Gamma(\alpha+1) \Gamma(\beta)} \int_{t_{k-2}}^{t_{k-1}}\left(t_{k-1}-s\right)^{\beta-1}\|f(s, u(s))-f(s, v(s))\| d s \\
& +|\lambda| \int_{t_{k}}^{t} \frac{(t-s)^{\alpha-1}}{\Gamma(\alpha)}\|u(s)-v(s)\| d s+|\lambda| \sum_{i=1}^{k} \int_{t_{i-1}}^{t_{i}} \frac{\left(t_{i}-s\right)^{\alpha-1}}{\Gamma(\alpha)}\|u(s)-v(s)\| d s \\
& +\frac{\left(t-t_{k}\right)^{\alpha}}{\Gamma(\alpha+1)} \sum_{i=1}^{k}\left\|\left(\lambda I_{i}+I_{i}^{*}\right)\left(u\left(t_{i}\right)\right)-\left(\lambda I_{i}+I_{i}^{*}\right)\left(v\left(t_{i}\right)\right)\right\| \\
& +\sum_{i=2}^{k} \frac{\left(t_{i}-t_{i-1}\right)^{\alpha}}{\Gamma(\alpha+1)}\left\|\left(\lambda I_{1}+I_{1}^{*}\right)\left(u\left(t_{1}\right)\right)-\left(\lambda I_{1}+I_{1}^{*}\right)\left(v\left(t_{1}\right)\right)\right\|+\cdots \\
& +\sum_{i=k-1}^{k} \frac{\left(t_{i}-t_{i-1}\right)^{\alpha}}{\Gamma(\alpha+1)}\left\|\left(\lambda I_{k-2}+I_{k-2}^{*}\right)\left(u\left(t_{k-2}\right)\right)-\left(\lambda I_{k-2}+I_{k-2}^{*}\right)\left(v\left(t_{k-2}\right)\right)\right\| \\
& +\frac{\left(t_{k}-t_{k-1}\right)^{\alpha}}{\Gamma(\alpha+1)}\left\|\left(\lambda I_{k-1}+I_{k-1}^{*}\right)\left(u\left(t_{k-1}\right)\right)-\left(\lambda I_{k-1}+I_{k-1}^{*}\right)\left(v\left(t_{k-1}\right)\right)\right\| \\
& \left.+\sum_{i=1}^{k}\left\|I_{i}\left(u\left(t_{i}\right)\right)-I_{i}\left(v\left(t_{i}\right)\right)\right\|\right\}
\end{aligned}
$$

Using the condition $\left(\mathrm{H}_{1}\right)$, by computation, we can get

$$
\begin{aligned}
& \|(T u)(t)-(T v)(t)\| \\
& \leq 2\left\{L_{1} \sum_{i=1}^{p+1} \int_{t_{i-1}}^{t_{i}} \frac{\left(t_{i}-s\right)^{\alpha-1}}{\Gamma(\alpha)} \int_{t_{i-1}}^{s} \frac{(s-r)^{\beta-1}}{\Gamma(\beta)} d r d s\|u-v\|_{P C}\right. \\
& +\frac{p L_{1}}{\Gamma(\alpha+1) \Gamma(\beta)} \int_{0}^{1}(1-s)^{\beta-1} d s\|u-v\|_{P C} \\
& +\frac{(p-1) L_{1}}{\Gamma(\alpha+1) \Gamma(\beta)} \int_{0}^{t_{1}}\left(t_{1}-s\right)^{\beta-1} d s\|u-v\|_{P C} \\
& +\cdots+\frac{2 L_{1}}{\Gamma(\alpha+1) \Gamma(\beta)} \int_{t_{k-3}}^{t_{k-2}}\left(t_{k-2}-s\right)^{\beta-1} d s\|u-v\|_{P C} \\
& +\frac{L_{1}}{\Gamma(\alpha+1) \Gamma(\beta)} \int_{t_{k-2}}^{t_{k-1}}\left(t_{k-1}-s\right)^{\beta-1} d s\|u-v\|_{P C} \\
& +|\lambda| \sum_{i=1}^{p+1} \int_{t_{i-1}}^{t_{i}} \frac{\left(t_{i}-s\right)^{\alpha-1}}{\Gamma(\alpha)} d s\|u-v\|_{P C} \\
& +\frac{p\left(|\lambda| L_{2}+L_{3}\right)}{\Gamma(\alpha+1)}\|u-v\|_{P C}+\frac{(p-1)\left(|\lambda| L_{2}+L_{3}\right)}{\Gamma(\alpha+1)}\|u-v\|_{P C} \\
& +\cdots+\frac{2\left(|\lambda| L_{2}+L_{3}\right)}{\Gamma(\alpha+1)}\|u-v\|_{P C}
\end{aligned}
$$




$$
\begin{aligned}
& \left.+\frac{\left(|\lambda| L_{2}+L_{3}\right)}{\Gamma(\alpha+1)}\|u-v\|_{P C}+p L_{2}\|u-v\|_{P C}\right\} \\
\leq & 2\left\{\frac{(p+1) L_{1}}{\Gamma(\alpha+1+\beta)}+\frac{L_{1}}{\Gamma(\alpha+1) \Gamma(\beta+1)} \sum_{i=1}^{p} i\right. \\
& \left.+\frac{(p+1)|\lambda|}{\Gamma(\alpha+1)}+\frac{\left(|\lambda| L_{2}+L_{3}\right)}{\Gamma(\alpha+1)} \sum_{i=1}^{p} i+p L_{2}\right\}\|u-v\|_{P C} \\
= & \left\{\frac{2(p+1) L_{1}}{\Gamma(\alpha+1+\beta)}+\frac{p(p+1) L_{1}}{\Gamma(\alpha+1) \Gamma(\beta+1)}\right. \\
& \left.+\frac{2(p+1)|\lambda|}{\Gamma(\alpha+1)}+\frac{p(p+1)\left(|\lambda| L_{2}+L_{3}\right)}{\Gamma(\alpha+1)}+2 p L_{2}\right\}\|u-v\|_{P C} .
\end{aligned}
$$

Thus, $\|T u-T v\|_{P C} \leq \Lambda\|u-v\|_{P C}$.

As $\Lambda<1$, therefore, $A$ is a contraction. Thus, the conclusion of the theorem follows by the contraction mapping principle.

\section{Nonlocal Dirichlet boundary value problems}

In this section, we consider the following nonlocal Dirichlet boundary value problems:

$$
\left\{\begin{array}{l}
{ }^{C} D^{\beta}\left({ }^{C} D^{\alpha}+\lambda\right) u(t)=f(t, u(t)), \quad 0<t<1,0<\alpha, \beta \leq 1, \\
\triangle u\left(t_{k}\right)=I_{k}\left(u\left(t_{k}\right)\right), \quad k=1,2, \ldots, p, \\
\triangle{ }^{C} D^{\alpha} u\left(t_{k}\right)=I_{k}^{*}\left(u\left(t_{k}\right)\right), \quad k=1,2, \ldots, p, \\
u(0)+g_{1}(u)=\gamma_{1}, \quad u(1)=\gamma_{2},
\end{array}\right.
$$

and

$$
\left\{\begin{array}{l}
{ }^{C} D^{\beta}\left({ }^{C} D^{\alpha}+\lambda\right) u(t)=f(t, u(t)), \quad 0<t<1,0<\alpha, \beta \leq 1, \\
\triangle u\left(t_{k}\right)=I_{k}\left(u\left(t_{k}\right)\right), \quad k=1,2, \ldots, p, \\
\triangle^{C} D^{\alpha} u\left(t_{k}\right)=I_{k}^{*}\left(u\left(t_{k}\right)\right), \quad k=1,2, \ldots, p, \\
u(0)=\gamma_{1}, \quad u(1)+g_{2}(u)=\gamma_{2} .
\end{array}\right.
$$

For the forthcoming analysis, we need the following assumptions:

$\left(\mathrm{H}_{2}\right)$ There exists a constant $L_{4}$ such that $\left\|g_{1}(u)-g_{1}(v)\right\| \leq L_{4}\|u-v\|$.

$\left(\mathrm{H}_{3}\right)$ There exists a constant $L_{5}$ such that $\left\|g_{2}(u)-g_{2}(v)\right\| \leq L_{5}\|u-v\|$.

Theorem 3.1 Assume $\left(\mathrm{H}_{1}\right),\left(\mathrm{H}_{2}\right)$ hold if $\Lambda+2 L_{4}<1$, then problem (3.1) has a unique solution, where $\Lambda$ is given by (2.14).

Theorem 3.2 Assume $\left(\mathrm{H}_{1}\right),\left(\mathrm{H}_{3}\right)$ hold if $\Lambda+L_{5}<1$, then problem (3.2) has a unique solution, where $\Lambda$ is given by (2.14).

The proofs of Theorem 3.2 and Theorem 3.1 are similar. Here we only prove Theorem 3.1. 
Proof We transform the problem (3.1) into a fixed point problem. Consider the operator $T_{1}: P C(J, E) \rightarrow P C(J, E)$ as follows:

$$
\begin{aligned}
T_{1} u(t)= & \int_{t_{k}}^{t} \frac{(t-s)^{\alpha-1}}{\Gamma(\alpha)} \int_{t_{k}}^{s} \frac{(s-r)^{\beta-1}}{\Gamma(\beta)} f(r, u(r)) d r d s \\
& +\sum_{i=1}^{k} \int_{t_{i-1}}^{t_{i}} \frac{\left(t_{i}-s\right)^{\alpha-1}}{\Gamma(\alpha)} \int_{t_{i-1}}^{s} \frac{(s-r)^{\beta-1}}{\Gamma(\beta)} f(r, u(r)) d r d s \\
& +\frac{\left(t-t_{k}\right)^{\alpha}}{\Gamma(\alpha+1) \Gamma(\beta)} \sum_{i=1}^{k} \int_{t_{i-1}}^{t_{i}}\left(t_{i}-s\right)^{\beta-1} f(s, u(s)) d s \\
& +\sum_{i=2}^{k} \frac{\left(t_{i}-t_{i-1}\right)^{\alpha}}{\Gamma(\alpha+1) \Gamma(\beta)} \int_{0}^{t_{1}}\left(t_{1}-s\right)^{\beta-1} f(s, u(s)) d s+\cdots \\
& +\sum_{i=k-1}^{k} \frac{\left(t_{i}-t_{i-1}\right)^{\alpha}}{\Gamma(\alpha+1) \Gamma(\beta)} \int_{t_{k-3}}^{t_{k-2}}\left(t_{k-2}-s\right)^{\beta-1} f(s, u(s)) d s \\
& +\frac{\left(t_{k}-t_{k-1}\right)^{\alpha}}{\Gamma(\alpha+1) \Gamma(\beta)} \int_{t_{k-2}}^{t_{k-1}}\left(t_{k-1}-s\right)^{\beta-1} f(s, u(s)) d s \\
& -\lambda \int_{t_{k}}^{t} \frac{(t-s)^{\alpha-1}}{\Gamma(\alpha)} u(s) d s-\lambda \sum_{i=1}^{k} \int_{t_{i-1}}^{t_{i}} \frac{\left(t_{i}-s\right)^{\alpha-1}}{\Gamma(\alpha)} u(s) d s \\
& +\frac{\left(t-t_{k}\right)^{\alpha}}{\Gamma(\alpha+1)} \sum_{i=1}^{k}\left(\lambda I_{i}+I_{i}^{*}\right)\left(u\left(t_{i}\right)\right)+\sum_{i=2}^{k} \frac{\left(t_{i}-t_{i-1}\right)^{\alpha}}{\Gamma(\alpha+1)}\left(\lambda I_{1}+I_{1}^{*}\right)\left(u\left(t_{1}\right)\right)+\cdots \\
& +\frac{C\left(t-t_{k}\right)^{\alpha}}{\Gamma(\alpha+1)}+C \sum_{i=1}^{k} \frac{\left(t_{i}-t_{i-1}\right)^{\alpha}}{\Gamma(\alpha+1)}+\gamma_{1}-g_{1}(u), \\
& +\sum_{i=k-1}^{k} \frac{\left(t_{i}-t_{i-1}\right)^{\alpha}}{\Gamma(\alpha+1)}\left(\lambda I_{k-2}+I_{k-2}^{*}\right)\left(u\left(t_{k-2}\right)\right) \\
& +\frac{\left.t_{k}-t_{k-1}\right)^{\alpha}}{\Gamma(\alpha+1)}\left(\lambda I_{k-1}+I_{k-1}^{*}\right)\left(u\left(t_{k-1}\right)\right)+\sum_{i=1}^{k} I_{i}\left(u\left(t_{i}\right)\right) \\
&
\end{aligned}
$$

where

$$
\begin{aligned}
C= & -\left[\sum_{i=1}^{p+1} \frac{\left(t_{i}-t_{i-1}\right)^{\alpha}}{\Gamma(\alpha+1)}\right]^{-1}\left\{\sum_{i=1}^{p+1} \int_{t_{i-1}}^{t_{i}} \frac{\left(t_{i}-s\right)^{\alpha-1}}{\Gamma(\alpha)} \int_{t_{i-1}}^{s} \frac{(s-r)^{\beta-1}}{\Gamma(\beta)} f(r, u(r)) d r d s\right. \\
& +\frac{\left(1-t_{p}\right)^{\alpha}}{\Gamma(\alpha+1) \Gamma(\beta)} \sum_{i=1}^{p} \int_{t_{i-1}}^{t_{i}}\left(t_{i}-s\right)^{\beta-1} f(s, u(s)) d s \\
& +\sum_{i=2}^{p} \frac{\left(t_{i}-t_{i-1}\right)^{\alpha}}{\Gamma(\alpha+1) \Gamma(\beta)} \int_{0}^{t_{1}}\left(t_{1}-s\right)^{\beta-1} f(s, u(s)) d s \\
& +\cdots+\sum_{i=p-1}^{p} \frac{\left(t_{i}-t_{i-1}\right)^{\alpha}}{\Gamma(\alpha+1) \Gamma(\beta)} \int_{t_{p-3}}^{t_{p-2}}\left(t_{p-2}-s\right)^{\beta-1} f(s, u(s)) d s \\
& +\frac{\left(t_{p}-t_{p-1}\right)^{\alpha}}{\Gamma(\alpha+1) \Gamma(\beta)} \int_{t_{p-2}}^{t_{p-1}}\left(t_{p-1}-s\right)^{\beta-1} f(s, u(s)) d s-\lambda \sum_{i=1}^{p+1} \int_{t_{i-1}}^{t_{i}} \frac{\left(t_{i}-s\right)^{\alpha-1}}{\Gamma(\alpha)} u(s) d s
\end{aligned}
$$




$$
\begin{aligned}
& +\frac{\left(1-t_{p}\right)^{\alpha}}{\Gamma(\alpha+1)} \sum_{i=1}^{p}\left(\lambda I_{i}+I_{i}^{*}\right)\left(u\left(t_{i}\right)\right)+\sum_{i=2}^{p} \frac{\lambda\left(t_{i}-t_{i-1}\right)^{\alpha}}{\Gamma(\alpha+1)}\left(\lambda I_{1}+I_{1}^{*}\right)\left(u\left(t_{1}\right)\right)+\cdots \\
& +\sum_{i=p-1}^{p} \frac{\left(t_{i}-t_{i-1}\right)^{\alpha}}{\Gamma(\alpha+1)}\left(\lambda I_{p-2}+I_{p-2}^{*}\right)\left(u\left(t_{p-2}\right)\right)+\frac{\left(t_{p}-t_{p-1}\right)^{\alpha}}{\Gamma(\alpha+1)}\left(\lambda I_{p-1}+I_{p-1}^{*}\right)\left(u\left(t_{p-1}\right)\right) \\
& \left.+\sum_{i=1}^{k} I_{i}\left(u\left(t_{i}\right)\right)+\gamma_{1}-g_{1}(u)-\gamma_{2}\right\} .
\end{aligned}
$$

The rest of the proof is almost the same as that of Theorem 2.1, so we omit it.

\section{Example}

The following example is a direct application of our main result.

Example 4.1 Consider the following Dirichlet boundary value problem of a nonlinear Langevin equation with two different fractional orders and impulses:

$$
\left\{\begin{array}{l}
{ }^{C} D^{\frac{3}{4}}\left({ }^{C} D^{\frac{1}{4}}-\frac{1}{20}\right) u(t)=\frac{\cos t}{(t+6)^{2}} \frac{\|u(t)\|}{1+\|u(t)\|}, \quad 0<t<1, t \neq \frac{1}{2}, \\
\triangle u\left(\frac{1}{2}\right)=\frac{\left\|u\left(\frac{1}{2}\right)\right\|}{8+\left\|u\left(\frac{1}{2}\right)\right\|}, \quad \Delta^{C} D^{\frac{1}{4}} u\left(\frac{1}{2}\right)=\frac{2\left\|u\left(\frac{1}{2}\right)\right\|}{20+\left\|u\left(\frac{1}{2}\right)\right\|}, \\
u(0)=\gamma_{1}, \quad u(1)=\gamma_{2},
\end{array}\right.
$$

where $\alpha=\frac{1}{4}, \beta=\frac{3}{4}, \lambda=-\frac{1}{20}, p=1, f(t, u)=\frac{\cos t}{(t+6)^{2}} \frac{\|u\|}{1+\|u\|}, I_{1}(u)=\frac{\|u\|}{8+\|u\|}$ and $I_{1}^{*}(u)=\frac{2\|u\|}{20+\|u\|}$.

Obviously, $L_{1}=\frac{1}{36}, L_{2}=\frac{1}{8}$ and $L_{3}=\frac{1}{10}$. Further,

$$
\begin{aligned}
\Lambda & =\frac{2(p+1) L_{1}}{\Gamma(\alpha+1+\beta)}+\frac{p(p+1) L_{1}}{\Gamma(\alpha+1) \Gamma(\beta+1)}+\frac{2(p+1)|\lambda|}{\Gamma(\alpha+1)}+\frac{p(p+1)\left(|\lambda| L_{2}+L_{3}\right)}{\Gamma(\alpha+1)}+2 p L_{2} \\
& \approx 0.882899<1 .
\end{aligned}
$$

Therefore, by Theorem 2.1, we can get that the above equation (4.1) has a unique solution on $[0,1]$.

\section{Competing interests}

The authors declare that they have no competing interests.

\section{Authors' contributions}

All authors completed the paper together. All authors read and approved the final manuscript.

\section{Author details}

${ }^{1}$ School of Mathematics and Computer Science, Shanxi Normal University, Linfen, Shanxi 041004, P.R. China.

2Department of Mathematics, China University of Petroleum, Qingdao, Shandong 266555, P.R. China.

\section{Acknowledgements}

We would like to express our gratitude to the anonymous reviewers and editors for their valuable comments and suggestions which have improved the quality of the present paper. The research was supported by the Natural Science Foundation for Young Scientists of Shanxi Province (2012021002-3), China.

Received: 15 March 2012 Accepted: 5 October 2012 Published: 6 November 2012

\section{References}

1. Kilbas, AA, Srivastava, HM, Trujillo, JJ: Theory and Applications of Fractional Differential Equations. North-Holland Mathematics Studies, vol. 204. Elsevier, Amsterdam (2006)

2. Podlubny, I: Fractional Differential Equations. Mathematics in Science and Engineering. Academic Press, New York (1999) 
3. Sabatier, J, Agrawal, OP, Machado, JAT (eds.): Advances in Fractional Calculus: Theoretical Developments and Applications in Physics and Engineering. Springer, Dordrecht (2007)

4. Ahmad, B, Nieto, Jj: Sequential fractional differential equations with three-point boundary conditions. Comput. Math. Appl. 64, 3046-3052 (2012)

5. Kaslik, E, Sivasundaram, S: Non-existence of periodic solutions in fractional-order dynamical systems and a remarkable difference between integer and fractional-order derivatives of periodic functions. Nonlinear Anal., Real World Appl. 13, 1489-1497 (2012)

6. Guezane-Lakoud, A, Khaldi, R: Solvability of a fractional boundary value problem with fractional integral condition. Nonlinear Anal. 75, 2692-2700 (2012)

7. Zhou, P, Zhu, W: Function projective synchronization for fractional-order chaotic systems. Nonlinear Anal., Real World Appl. 12, 811-816 (2011)

8. Goodrich, CS: Existence of a positive solution to systems of differential equations of fractional order. Comput. Math. Appl. 62, 1251-1268 (2011)

9. Agarwal, RP, Benchohra, M, Hamani, S, Pinelas, S: Boundary value problems for differential equations involving Riemann-Liouville fractional derivative on the half-line. Dyn. Contin. Discrete Impuls. Syst. Ser. A Math. Anal. 18, 235-244 (2011)

10. Baleanu, D, Agarwal, RP, Mustafa, OG, Cosulschi, M: Asymptotic integration of some nonlinear differential equations with fractional time derivative. J. Phys. A 44, Article ID 055203 (2011)

11. Agarwal, RP, Zhou, Y, Wang, J, Luo, X: Fractional functional differential equations with causal operators in Banach spaces. Math. Comput. Model. 54, 1440-1452 (2011)

12. Wang, G, Agarwal, RP, Cabada, A: Existence results and the monotone iterative technique for systems of nonlinear fractional differential equations. Appl. Math. Lett. 25, 1019-1024 (2012)

13. Chen, F, Nieto, JJ, Zhou, Y: Global attractivity for nonlinear fractional differential equations. Nonlinear Anal., Real World Appl. 13, 287-298 (2012)

14. Wang, J, Zhou, Y: A class of fractional evolution equations and optimal controls. Nonlinear Anal. 12, 262-272 (2011)

15. Wang, G, Ntouyas, SK, Zhang, L: Positive solutions of the three-point boundary value problem for fractional-order differential equations with an advanced argument. Adv. Differ. Equ. 2011, 2 (2011)

16. Wax, N (ed.): Selected Papers on Noise and Stochastic Processes. Dover, New York (1954)

17. Mazo, R: Brownian Motion: Fluctuations, Dynamics and Applications. Oxford Univ. Press, Oxford (2002)

18. Coffey, WT, Kalmykov, YP, Waldron, JT: The Langevin Equation, 2nd edn. World Scientific, Singapore (2004)

19. Kobolev, V, Romanov, E: Fractional Langevin equation to describe anomalous diffusion. Prog. Theor. Phys. Suppl. 139, 470-476 (2000)

20. Lim, SC, Muniandy, SV: Self-similar Gaussian processes for modeling anomalous diffusion. Phys. Rev. E 66, 021114 (2002)

21. Picozzi, S, West, B: Fractional Langevin model of memory in financial markets. Phys. Rev. E 66, 046118 (2002)

22. Lim, SC, Li, M, Teo, LP: Locally self-similar fractional oscillator processes. Fluct. Noise Lett. 7, 169-179 (2007)

23. Lim, SC, Li, M, Teo, LP: Langevin equation with two fractional orders. Phys. Lett. A 372(42), 6309-6320 (2008)

24. Lim, SC, Teo, LP: The fractional oscillator process with two indices. J. Phys. A 42(6), Article ID 065208 (2009)

25. Ahmad, B, Nieto, JJ: Solvability of nonlinear Langevin equation involving two fractional orders with Dirichlet boundary conditions. Int. J. Differ. Equ. 2010, Article ID 649486 (2010)

26. Ahmad, B, Nieto, JJ, Alsaedi, A, El-Shahed, M: A study of nonlinear Langevin equation involving two fractional orders in different intervals. Nonlinear Anal., Real World Appl. 13, 599-606 (2012)

27. Byszewski, L, Lakshmikantham, V: Theorem about the existence and uniqueness of a solution of a nonlocal abstract Cauchy problem in a Banach space. Appl. Anal. 40, 11-19 (1991)

28. Byszewski, L: Theorems about existence and uniqueness of solutions of a semilinear evolution nonlocal Cauchy problem. J. Math. Anal. Appl. 162, 494-505 (1991)

29. Balachandran, K, Trujillo, JJ: The nonlocal Cauchy problem for nonlinear fractional integrodifferential equations in Banach spaces. Nonlinear Anal. 72, 4587-4593 (2010)

30. N'Guerekata, GM: A Cauchy problem for some fractional abstract differential equation with nonlocal condition. Nonlinear Anal. 70, 1873-1876 (2009)

31. Feng, $M$, Zhang, $X, G$, W: New existence results for higher-order nonlinear fractional differential equation with integral boundary conditions. Bound. Value Probl. 2011, art. no. 720702 (2011)

32. Salem, HAH: Fractional order boundary value problem with integral boundary conditions involving Pettis integral. Acta Math. Sci. 31, 661-672 (2011)

33. Ahmad, B, Nieto, JJ, Alsaedi, A: Existence and uniqueness of solutions for nonlinear fractional differential equations with non-separated type integral boundary conditions. Acta Math. Sci. 31, 2122-2130 (2011)

34. Ahmad, B, Nieto, Jj: Existence results for nonlinear boundary value problems of fractional integrodifferential equations with integral boundary conditions. Bound. Value Probl. 2009, art. no. 708576 (2009)

35. Ahmad, B, Ntouyas, SK, Alsaedi, A: New existence results for nonlinear fractional differential equations with three-point integral boundary conditions. Adv. Differ. Equ. 2011, art. no. 107384 (2011)

36. Benchohra, M, Graef, JR, Hamani, S: Existence results for boundary value problems with nonlinear fractional differential equations. Appl. Anal. 87, 851-863 (2008)

37. Hamani, S, Benchohra, M, Graef, JR: Existence results for boundary value problems with nonlinear fractional inclusions and integral conditions. Electron. J. Differ. Equ. 20, 1-16 (2010)

38. Liu, X, Jia, M, Wu, B: Existence and uniqueness of solution for fractional differential equations with integral boundary conditions. Electron. J. Qual. Theory Differ. Equ. 69, 1-10 (2009)

39. Cabada, A, Wang, G: Positive solutions of nonlinear fractional differential equations with integral boundary value conditions. J. Math. Anal. Appl. 389, 403-411 (2012)

40. Benchohra, M, Hamani, S, Ntouyas, SK: Boundary value problems for differential equations with fractional order and nonlocal conditions. Nonlinear Anal. 71, 2391-2396 (2009)

41. Wang, G, Ahmad, B, Zhang, L: New existence results for nonlinear impulsive integro-differential equations of fractional order with nonlocal boundary conditions. Nonlinear Stud. (to appear) 
42. Wang, G, Ahmad, B, Zhang, L: Some existence results for impulsive nonlinear fractional differential equations with mixed boundary conditions. Comput. Math. Appl. 62, 1389-1397 (2011)

43. Wang, G, Ahmad, B, Zhang, L: Impulsive anti-periodic boundary value problem for nonlinear differential equations of fractional order. Nonlinear Anal. 74, 792-804 (2011)

44. Zhang, L, Wang, G: Existence of solutions for nonlinear fractional differential equations with impulses and anti-periodic boundary conditions. Electron. J. Qual. Theory Differ. Equ. 2011, 7 (2011)

45. Ahmad, B, Wang, G: A study of an impulsive four-point nonlocal boundary value problem of nonlinear fractional differential equations. Comput. Math. Appl. 62, 1341-1349 (2011)

46. Ahmad, B, Nieto, J: Existence of solutions for impulsive anti-periodic boundary value problems of fractional order. Taiwan. J. Math. 15, 981-993 (2011)

47. Ahmad, B, Sivasundaram, S: Existence of solutions for impulsive integral boundary value problems of fractional order Nonlinear Anal. Hybrid Syst. 4, 134-141 (2010)

48. Agarwal, RP, Ahmad, B: Existence of solutions for impulsive anti-periodic boundary value problems of fractional semilinear evolution equations. Dyn. Contin. Discrete Impuls. Syst. Ser. A Math. Anal. 18, 457-470 (2011)

49. Mophou, GM: Existence and uniqueness of mild solutions to impulsive fractional differential equations. Nonlinear Anal. 72, 1604-1615 (2010)

50. Tian, Y, Bai, Z: Existence results for the three-point impulsive boundary value problem involving fractional differential equations. Comput. Math. Appl. 59, 2601-2609 (2010)

51. Zhang, $X$, Huang, $X$, Liu, Z: The existence and uniqueness of mild solutions for impulsive fractional equations with nonlocal conditions and infinite delay. Nonlinear Anal. Hybrid Syst. 4, 775-781 (2010)

doi:10.1186/1687-1812-2012-200

Cite this article as: Wang et al.: Boundary value problem of a nonlinear Langevin equation with two different

fractional orders and impulses. Fixed Point Theory and Applications 2012 2012:200.

\section{Submit your manuscript to a SpringerOpen ${ }^{\ominus}$ journal and benefit from:}

- Convenient online submission

- Rigorous peer review

- Immediate publication on acceptance

Open access: articles freely available online

- High visibility within the field

- Retaining the copyright to your article 